\title{
ON A CONJECTURE CONCERNING DOUBLY STOCHASTIC MATRICES
}

S. SHERMAN

A doubly stochastic (d.s.) matrix is a real $n \times n$ matrix $P=\left(p_{i j}\right)$ such that

$$
\begin{aligned}
p_{i j} \geqq 0, & 1 \leqq i \leqq n, 1 \leqq j \leqq n, \\
\sum_{j} p_{i j}=1, & 1 \leqq i \leqq n,
\end{aligned}
$$

and

$$
\sum_{i} p_{i j}=1
$$$$
1 \leqq j \leqq n .
$$

We introduce a partial order among d.s. matrices by defining

$$
P^{1} \prec P^{3}
$$

to mean there exists a d.s. matrix $P^{2}$ such that

$$
P^{1}=P^{2} P^{3} \text {. }
$$

We introduce a partial order among real vectors $a=\left(a_{1}, \cdots, a_{n}\right)$ of our real $n$-dimensional space $E$ by defining

$$
a<b
$$

to mean for each real convex $\phi$

$$
\sum_{i} \phi\left(a_{i}\right) \leqq \sum_{i} \phi\left(b_{i}\right) .
$$

By [HLP, p. 89], $a \prec b$ if and only if there exists a d.s. matrix $P$ such that

$$
a=P b=\left(\sum_{j} p_{1 j} b_{j}, \cdots, \sum_{i} p_{n} b_{i}\right) .
$$

This implies that for each real $n$-vector $a$,

$$
P^{1}<P^{3} \rightarrow P^{1} a<P^{3} a .
$$

Kakutani has raised the following conjecture.

Conjecture. If, for each real $n$-vector $a, P^{1} a<P^{3} a$, then $P^{1}<P^{3}$. By [HLP, p. 89] if the hypothesis is satisfied there exists a d.s.

Presented to the Society, December 28, 1951; received by the editors November $19,1951$. 
matrix $P_{a}^{2}$ such that

$$
P^{1} a=P_{a}^{2} P^{3} a .
$$

The issue is to show that if there exists such a $P_{a}^{2}$ for each vector $a$, there exists a d.s. $P^{2}$ independent of $a$ such that $P^{1}=P^{2} P^{3}$.

Let $P$ be the collection of vectors with non-negative components. Requirement (1) is equivalent to the requirement

$$
P P \subset P \text {. }
$$

If $e$ is the vector all of whose components are unity, then requirement (2) is equivalent to requirement

$$
P e=e,
$$

i.e., $e$ is a characteristic vector of characteristic value unity. If $\bar{e}$ is the element of $\bar{E}$, the conjugate space of $E$, whose value at $a \in E$ is given by $(\bar{e}, a)=\sum_{i} a_{i}$, then $\bar{e}^{\perp}=\{a \mid(\bar{e}, a)=0\}$ is the set of vectors $a \in E$ whose components add up to zero. Requirement (3) is equivalent to

$$
P\left(\bar{e}^{\perp}\right) \subset \bar{e}^{\perp}
$$

Proof of conjecture. Suppose now $P^{1} a<P^{3} a$ for each $a \in E$. Consider a mapping $\psi: P^{3} E \rightarrow P^{1} E$ defined as follows: if $b \in P^{3} E$, for some $a \in E$ we have $b=P^{3} a$, then let $\psi(b)=P^{1} a$. We first prove (i) that we have a valid definition, i.e., $\psi(b)$ is uniquely defined by the above and then we prove (ii) that $\psi: P^{3} E \rightarrow P^{1} E$ is a linear transformation.

In order to prove (i) suppose that $P^{3} a^{\prime}=P^{3} a^{\prime \prime}=b$. We wish to show that $P^{1} a^{\prime}=P^{1} a^{\prime \prime}$. If $P^{3} a^{\prime}=P^{3} a^{\prime \prime}$, then $P^{3}\left(a^{\prime}-a^{\prime \prime}\right)=0$, the zero vector. Since $P^{1}\left(a^{\prime}-a^{\prime \prime}\right)<P^{3}\left(a^{\prime}-a^{\prime \prime}\right)$, by (9) we deduce that $P^{1}\left(a^{\prime}-a^{\prime \prime}\right)=0$ and so $P^{1} a^{\prime}=P^{1} a^{\prime \prime}$. Thus we have shown that $\psi$ is uniquely defined. The linearity (ii) of $\psi$ is now trivial. If $\alpha$ is a real scalar and $P^{3} a=b$, then $P^{3} \alpha a=\alpha b$ and $\psi(\alpha b)=P^{1} \alpha a=\alpha P^{1} a=\alpha \psi(b)$. Also if $P^{3} a^{\prime}=b^{\prime}$ and $P^{3} a^{\prime \prime}=b^{\prime \prime}$, then $P^{3}\left(a^{\prime}+a^{\prime \prime}\right)=b^{\prime}+b^{\prime \prime}$ and $\psi\left(b^{\prime}+b^{\prime \prime}\right)=P^{1}\left(a^{\prime}+a^{\prime \prime}\right)$ $=P^{1} a^{\prime}+P^{1} a^{\prime \prime}=\psi\left(b^{\prime}\right)+\psi\left(b^{\prime \prime}\right)$.

Suppose $P^{3} a \in P$. Since $P^{1} a<P^{3} a$ by (9) there exists a d.s. $P_{a}$ such that $P^{1} a=P_{a} P^{3} a \in \mathcal{P}$. Thus $\psi\left(P \cap P^{3} E\right) \subset \mathcal{C}$. Since $P^{1}$ and $P^{3}$ are d.s. matrices, $P^{1} e=P^{3} e=e$ and so $\psi(e)=e$. If by $(P a)_{i}$ we denote the $i$ th component of $P a$, then by [HLP, p. 89] and the assumption $P^{1} a$ $<P^{3} a$ we have

$$
\sum_{i}\left(P^{1} a\right)_{i}=\sum_{i}\left(P^{3} a\right)_{i}
$$


In particular

$$
\psi\left(\bar{e}^{\perp} \cap P^{3} E\right) \subset \bar{e}^{\perp} .
$$

We can now extend $\psi$ to a function $\Psi$ on all of $E$ by letting $\Psi(b)$ $=0$ for each $b \in E \cap\left(P^{3} E\right)^{\prime}$, the complement of $P^{3} E$, and $\Psi(b)=\psi(b)$ for each $b \in P^{3} E$. Now $\Psi$ is a linear transformation satisfying the requirements

$$
\Psi(P) \subset \mathcal{P}, \quad \Psi(e)=e, \quad \Psi\left(\bar{e}^{\perp}\right) \subset \bar{e}^{\perp} .
$$

Therefore we can represent $\Psi$ by a d.s. matrix $P^{2}$. We now have $P^{1} a$ $=P^{2} P^{3} a$ for each $a \in E$ and therefore $P^{1}=P^{2} P^{3}$, thus establishing the conjecture.

It can readily be shown that if $P^{1}<P^{3}$, then for each $j=1,2, \cdots, n$

$$
\left(p_{1 j}^{1}, p_{2 j}^{1}, \cdots, p_{n j}^{1}\right)<\left(p_{1 j}^{3}, \cdots, p_{n j}^{3}\right) .
$$

It would be interesting to establish the converse.

\section{REFERENCE}

G. H. Hardy, J. E. Littlewood, and G. Polya, Inequalities, Cambridge University Press, 1934.

Navy Research Group, Lockheed Aircraft Corporation 